\title{
Complications of carotid angioplasty and stenting
}

\section{Jacques Théron, M.D., Léopoldo Guimaraens, M.D., Oguzman Coskun, Thérésa Sola, M.D., Jean-Baptiste Martin, M.D., and Daniel A. Rüfenacht, M.D.}

University Hospital, Caen, France; Hospital General de Cataluña, Barcelona, Spain; and University Hospital of Geneva, Geneva, Switzerland[

The authors report the complications that occurred in their experience with performing recanalization procedures in the internal carotid artery and present their treatment strategies. The complications can be classified into those that were periprocedural and those that were postprocedural. The former include complications related to the vascular-approach access site of and those associated with the dilation and stenting procedure. Other complications observed included embolic events, dissection, vascular spasm, bradycardia, inappropriate dilation, occlusion of the external carotid artery, and rare, unusual complications such as the occurrence of iatrogenic cavernous carotid fistula. Postprocedure complications occurred in the hours and days following the procedure in the form of embolic and occlusive events, and hypotension and bradycardia were seen as late complications in the months following the procedure. The authors discuss how such complications occur and provide suggestions on how to avoid them. The role of stent placement and the potential use of protective devices are explored. Overall, adequate use of currently available systems allows for safe application of endovascular treatment techniques that avoid altogether or treat these potential complications. A reduced incidence of complications related to the initial individual learning curve may be obtained with preclinical training, in which use of invitro models should be considered. Surgical standby no longer seems required; however, early posttreatment surveillance in intensive care unit is mandatory to avoid the remaining primary complications.

Key Words * carotid artery * atheromatous stenosis * stent * complication * cerebral protection

As a minimally invasive treatment option for atheromatously diseased carotid bifurcation stenoses, carotid artery angioplasty and stenting is becoming an increasingly popular procedure. Still considered a controversial procedure, carotid artery angioplasty and stenting will go through a period in which it is critically evaluated and compared with the well-established surgical carotid endarterectomy procedure before it becomes more uniformly accepted. In our opinion, a breakthrough is expected only if the complication rate of the endovascular minimally invasive procedure is not only comparable but significantly lower than the known complication rates of the surgical endarterectomy procedures. This will require a further reduction in the currently reported complication rates of endovascular procedures. 
This also will require that surgeons possess a good knowledge of the potential complications, the capability to manage them, and efforts to avoid them. Appropriate training to recognize dangerous conditions and to react adequately and with competence appears mandatory. Because of the current rate of dissemination of endovascular technology, development of training conditions and definition of optimum requirements is critical to train the number of future specialists necessary to care for patients with atheromatously diseased carotid bifurcation stenoses who may benefit from minimally invasive treatment options.

\section{COMPLICATIONS RELATED TO THE SITE OF VASCULAR APPROACH}

\section{Femoral Approach and General Considerations}

Since Seldinger introduced the technique for percutaneous catheter placement and due to evolving catheter technology, the femoral approach has gained so much popularity that it is now widely recognized as the optimum endovascular access site when treating craniocervical arteries. Its major advantages include the possibility to select and reach from a single puncture site all craniocervical arteries, which allows for treatment of one or several vascular segments and for angiographic exploration of the whole cerebral vascular system. In addition, greater patient safety and comfort are associated with this procedure, allowing for examination after induction of a local anesthesic. This puncture site may be kept at a distance from the area being treated and this avoids potential complications. Working from the patient's groin provides a critical increase in distance from the previously irradiated site, thereby allowing medical personnel to avoid the cumulative effects of radiation. Indeed, with correct use of radiological installation and radiation protection measures, the risk of undue radiation is kept low, both for the patient and for medical personnel.

The safe application of this technique to the "femoral approach," however, requires some training to overcome difficulties related mostly to the complex and tortuous vascular anatomy. Surgeons who are experienced at performing surgery in carotid bifurcations and who may utilize endovascular techniques in the future are advised to be appropriately trained in the technique of supraaortic vessel catheterization. For example, when treating the left carotid artery bifurcation via a femoral approach through a tortuous left common carotid artery (CCA) in an 83-year-old neurologically symptomatic patient who presents with general vascular atheromatosis, including stenotic and tortuous femoral and iliac atheromatous disease, the need to position safely the endovascular instruments provides inherent technical difficulties and complications that were faced and essentially overcome more than 25 years ago using neuroradiological angiographic techniques.

\section{Femoral Approach for Carotid Artery Stenting}

Currently used devices for carotid artery stenting, such as the Wallstent (Schneider Europe and Boston Scientific Corporation, Bülach, Switzerland), have dimensions that require a minimal lumen that is provided by a No. 7 French introducer sheath or a No. 9 French guide catheter. Therefore, after puncture, the femoral artery approach will be dilated to a hole of 3 to $3.6-\mathrm{mm}$ size, the equivalent of a No. 7 to No. 9 French introducer sheath. Currently we prefer the use of No. 9 French introducer sheaths because they allow continued use of the classic Wallstent "rolling membrane" type. This, after early experiences with the "Easy Wallstent" have revealed limits and complications that will be discussed.

After gaining femoral access and when the introducer sheath has been positioned and attached to the skin, full heparinization therapy is begun because it poses little risk of bleeding at the puncture site. 
We recommend withdrawing the introducer sheath in the morning after surgery. This avoids reversal of anticoagulation, while continuous administration of antiplatelets (ticlopidine and aspirin) is performed. After withdrawal of the introducer sheath, and adequate compression has been applied, there remains a risk of hematoma formation, particularly if withdrawl is performed too early after the procedure while the patient's hemostasis is still influenced by heparin and the concomitantly administered antiplatelet therapy. An additional factor that likely causes hematoma formation is a noncompliant, nervous patient, who bends the hip too early and thereby places the initially successful hemostasis at risk of rebleeding. If early withdrawal is mandated to avoid progressive ischemic symptoms of a leg due to preexisting critical blood supply, then the use of the Femostop II hemostatic device (Radi Medical Systems, Uppsala, Sweden) or Angio-Seal (Sherwood Davis \& Geck, St. Louis, MO) is recommended. As already indicated, we encountered the most satisfactory situation when withdrawing the introducer sheath the morning after surgery when the patient was calm and stable.

Delayed femoral hematomas may also occur and, in our experience, have been associated with patients who undergo treatment with ticlopidine. Rarely, surgical removal of the hematoma will be required. Pseudoaneurysm formation can be addressed first by using sonographically controlled compression, which is likely to initiate thrombosis of the aneurysmal cavity.

The femoral approach may be chosen even in cases in which the patient has received a femoropopliteal graft. If the graft is directly punctured or if a large introducer sheath is used, surgical repair after induction of a local anesthesic will be required; in our opinion, this is preferable to the alternative option of performing a direct neck approach after induction of a general anesthesic, which is associated with a higher incidence of risk.

\section{Carotid (Neck) Approach for Carotid Artery Stenting}

The neck approach with direct puncture of the CCA should be limited to use in patients in whom the femoral approach is prohibited, mainly because of extended occlusive iliac or femoral atheromatous vascular lesions.

The direct carotid artery puncture is associated with limitations and risks related to local anatomical conditions. There is a risk of plaque detachment and dissection, with the consequent potential of thromboembolic events. Access at this level limits the possibility of angiographic studies to the vessel segments that are distal to the puncture site. If angiographic control of other vascular territories is required, additional vascular access must be obtained. Another disadvantage, manipulation in proximity of the irradiated field must be considered. However, the main problem in our experience lies in the risk of hematoma formation, with the potential of mass effect in the upper airways. The fear of such hematoma formation will lead to the use of a reduced and consequently less secure heparinization. There is need for general anesthesia to avoid inadvertent head movements and to allow for safe removal of the introducer sheath, thus averting the significant risk of hematoma formation in the neck (even if the heparinization has been reversed). The immediate removal of the introducer sheath requires establishment of an additional arterial line (radial artery) to maintain continuous monitoring of arterial pressure values in the early posttreatment period.

The advantage of a direct carotid artery access is limited by the facility with which direct manipulation of medical devices is possible. For understandable reasons, this is the most familiar access site for carotid artery stenting currently favored by some surgeons. The direct carotid artery approach should be limited to patients with otherwise very restricted vascular access, due to previously stated risks. 


\section{Axillary Approach for Carotid Artery Stenting}

Another alternative approach is the right axillary approach, which provides access to the right or left carotid artery. Selective catheterization is performed by using a catheter with a standard tip shape. If the arm approach is chosen for treatment with stent implantation, which requires the use of a large-sized introducer sheath, a high brachial arterial puncture is favored. This as a good compromise between either the axillary artery puncture, where hematoma formation is difficult to control, or the low brachial approach, where there is significant ischemic risk to the hand due to potential vasospasm and thrombus formation.

\section{COMPLICATIONS DURING DILATION AND STENTING}

\section{Embolic Complications}

Where do Emboli Come From? Embolic events are by far the most frequent complications encountered in recanalization procedures. Embolic events may be related to injection or release of clot, fibrin, air, or plaque debris. If emboli occur in vessels that supply the brain, they may produce dramatic neurological or neuropsychological deficits. Because they may occur in the course of any type of diagnostic or therapeutic endovascular procedure, they are the most feared complications when performing diagnostic angiography. Subsequently, only the embolic complications directly related to the angioplasty and stenting procedure will be addressed, because the other embolic complications related to the endovascular approach may be avoided by basic angiographic training.

Because the potential exists to produce embolic events when treating carotid artery stenotic lesions, several specific aspects must be considered. Stenotic lesions at the carotid bifurcation are variable depending on the level and type of disease involved. The incidence of embolic risk can be considered as low in treating nonatheromatous lesions. In atheromatous lesions, the embolic risk likewise can be considered low when innominate trunk, proximal CCA, or distal ICAs are treated, because all of these sites rarely appear with aspects of ulcers. The embolic risk in these locations is presumably comparable to that in subclavian artery angioplasty.

However, atheromatous stenotic lesions at the bifurcation are quite different because the plaque characteristically demonstrates proliferative, fragile, and frequently ulcerated lesions. We know from surgical observations and from pathological specimens about this aspect of the carotid bifurcation plaque and have learned from initial personal and reported experience about the reality of this potential to produce plaque debris and clot with or without dissection; therefore, we decided to be safe by applying techniques that provide protection for cerebral arteries. Our belief that angioplasty and stent use increases the risk of thromboembolic events during the procedure has been corrobated by scientific support recently presented in research data that confirms the abundance of plaque debris produced with current technology.[5,7] Furthermore, it is our understanding that release of plaque debris is not prevented by current stent technology because the mesh size is too large compared with the debris size. It is more likely that the stent produces debris by shaving it off while the struts move against the vessel lumen surface. This occur mostly during the postdilation procedure. Therefore, we consider the postdilation procedure the most dangerous stage with regard to producing embolic events.

Although less likely, plaque debris release could occur during the first passage through a tight and irregular stenotic lesion, with the treatment devices irritating the plaque. However, it is our observation that during this first passage, a wedge position of the catheter system in the stenotic carotid segment is 
obtained, thereby producing flow arrest and preventing embolism. If a protection system is deployed prior to reestablishment of flow, then embolic events should not be feared, even from the first passage of instruments through the stenotic area.[8] If gentle predilation is required, the risk of embolic events also appears low. The risk of producing clot is enhanced if insufficient anticoagulation therapy is performed and dissection occurs.

\section{How Cerebral Protection Evolved and How to Treat Embolic Complications-Personal Experience.}

According to our initial experience, there is approximately an $8 \%$ risk of complications when angioplasty is performed without cerebral protection measures. Because no correlation with plaque morphology was possible and because embolic events also occur in apparently soft and smooth stenoses, the prediction of embolic events is difficult. We would like to stress that this occurred at approximately the same rate as reported in series in which direct stenting[1-4,6,8] was performed without cerebral protection measures, confirming that the stent has no protective effect against cerebral thromboembolism. Cerebral protection and use of cleaning with aspiration and flush during dilation procedures has allowed us to avoid and dramatically reduce embolic complications.[2]

With initial stent use to avoid problems related to dissection, it was technically not possible to provide simultaneous protection. Therefore we placed Strecker stents without protection after performing protected angioplasty, and we observed an embolic complication rate of $2.5 \%$; fortunately all were successfully dissolved (Fig. 1).
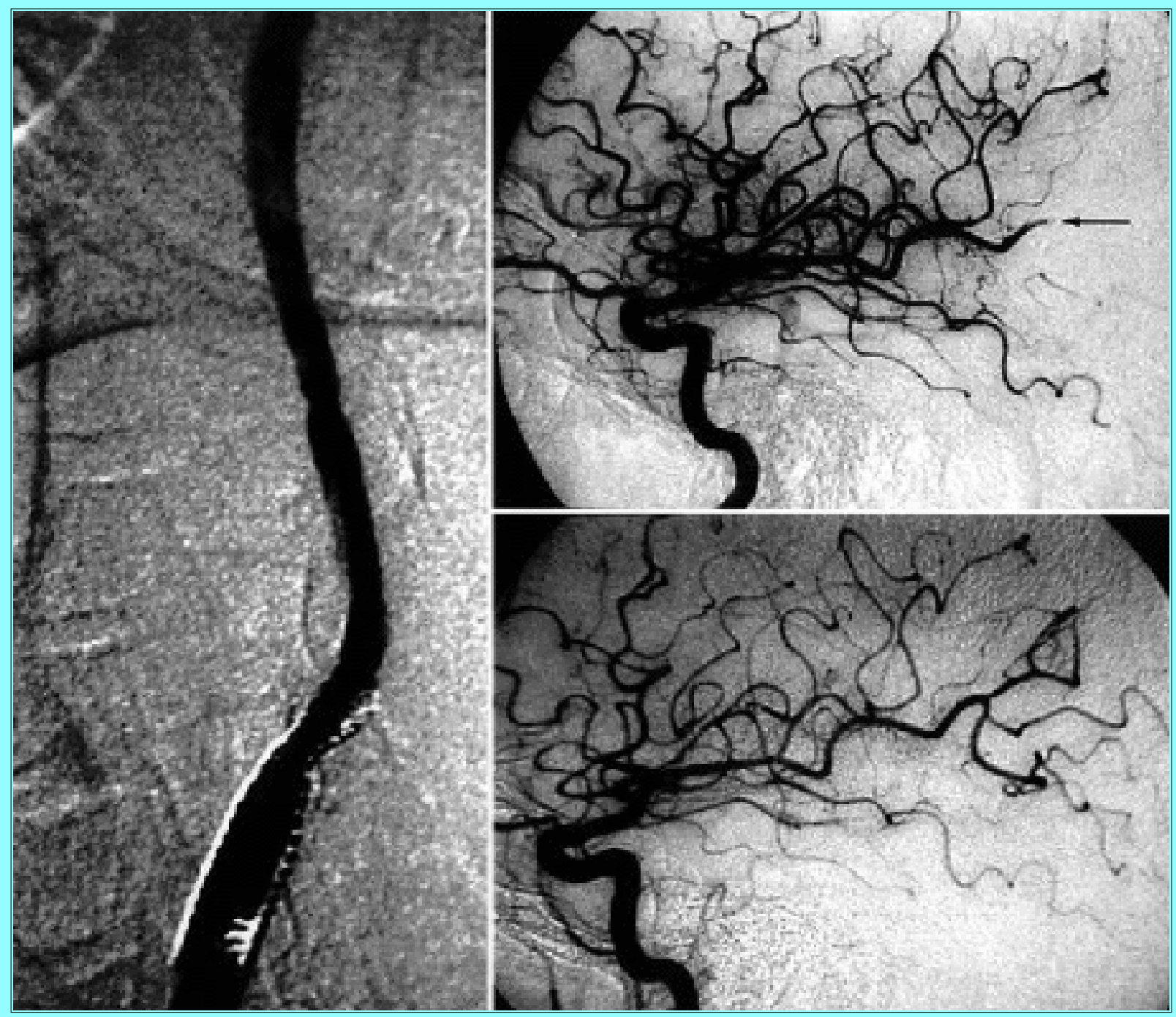
Fig. 1. Angiographic studies exemplifying thrombus formation in the carotid artery during stent placement associated with intracerebral thromboembolus. Intraarterial thrombolysis was performed. Left: Angiogram demonstrating clot formation in the stent positioned in the CCA and ICA and occlusion of the ECA. Upper Right: Cerebral angiogram, lateral projection showing embolic occlusion of a posterior cortical branch of the middle cerebral artery. The patient sustained related neurological deficit of the contralateral hemibody. Lower Right: Angiogram obtained immediately after intraarterial thrombolysis (with urokinase) showing opening of the occluded cortical branches. The patient recovered completely from the neurological deficit.

With the introduction of Wallstents, which exhibit a large lumen size for the stent delivery device and allow for use of a protective measures during stent implantation, embolic complications again became rare.

Intraarterial Thrombolysis. Although intraarterial use of thrombolytic therapy may allow for clot dissolution in an efficient way, plaque debris is not influenced by this treatment. Nevertheless, potentially associated clots can be dissolved by use of thromoblytic therapy. Indications for use of intraarterial thrombolysis exist in the following three typical circumstances. 1) Preventively, use of thrombolysis can be considered prior to angioplasty procedure, when the plaque surface appears ulcerated and irregular. The thrombolytic drug (r-TPA $20 \mathrm{mg}$ or 300,000 IU of urokinase) is injected either directly into the ICA or, even more efficiently, after temporary distal occlusion with a protection device to allow for increased local drug exposure. The adherent clot is thus dissolved or can be washed away by aspiration and flush after a few minutes (Fig. 2).
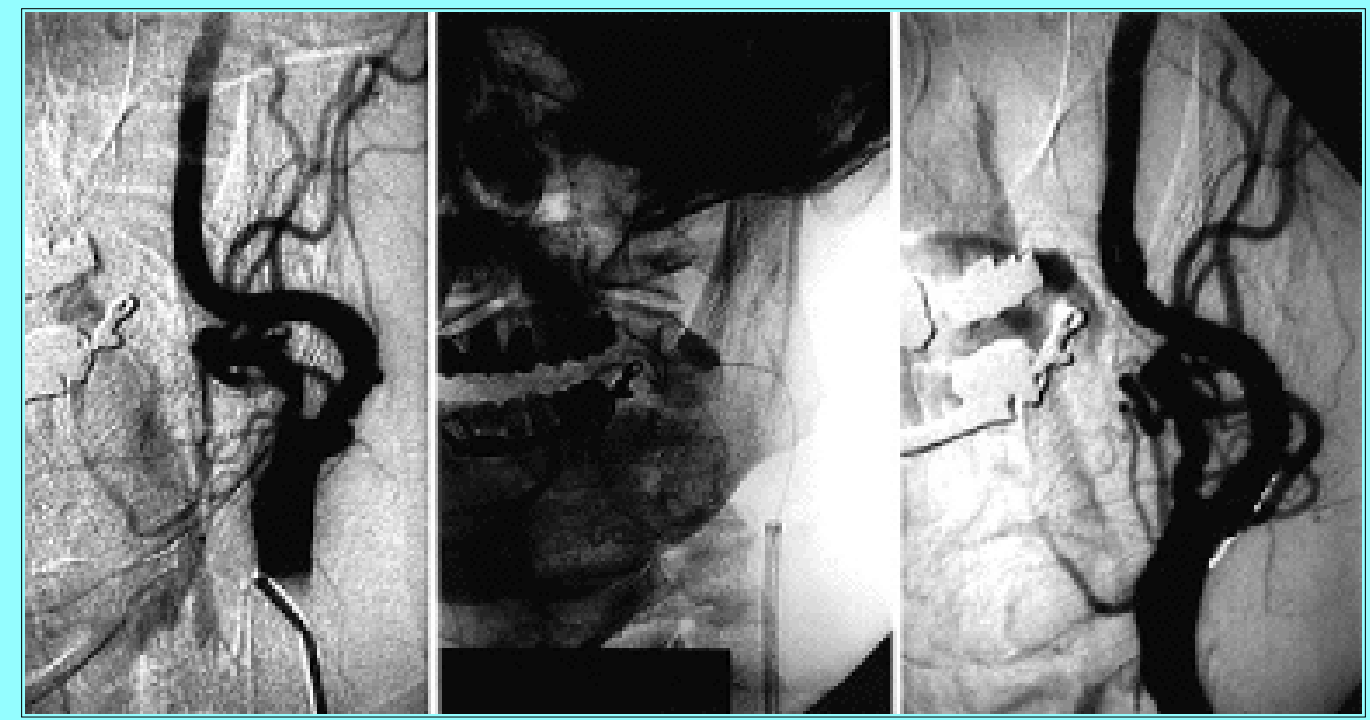

Fig. 2. Angiographic studies demonstrating preventive intraarterial thrombolysis before a nonprotected stenting procedure and the presence of an ulcerated symptomatic stenosis. Left: Angiogram obtained before stent placement showing irregularities in the arterial wall with ulcerations. Center: Temporary occlusion of the ICA. Injection of 300,000 IU of urokinase in the ICA below the occlusion balloon through the guiding catheter. After 3 minutes the blood was aspirated prior to performing protected angioplasty and unprotected placement of a Strecker stent. Right: Control angiogram posttreatment.

During a prolonged procedure, in cases in which the approach has been difficult because of very tortuous vessels, it is mandatory to reinject heparin, because there is a high risk of clot formation on freshly 
introduced metallic implants, such as a stent. Endoluminal filling defects, which indicate clot formation on the stent, may be lysed with immediate adminstration of the local intraarterial thrombolytic agent (Fig. 3). If a thromboembolic complication in the cerebral arteries occurs, as visualized on control angiography, and is clinically symptomatic, or both, thrombolysis should be considered, although embolic occlusions may be due to plaque debris only. It is our belief that thrombolysis performed for this indication may improve local flow conditions by dissolving the clot induced by plaque debris, thereby increasing the chances to reduce the size of the infarction. We currently use for this indication r-TPA at a dose of $40 \mathrm{mg}$ or urokinase up to the total of 1,000,000 IU slowly injected for half the dose in the ICA followed by half the dose delivered superselectively close to the embolus in the middle cerebral artery. The total drug dose should be administered immediately and within 1 to 2 hours after onset of the symptoms and should not exceed the equivalent of approximately 500,000 IU/hour. Superselective drug delivery is performed only by competent, trained medical personnel to avoid additional risks. In situations in which superselective catheterization is not available, we propose injecting the full dose of thrombolytic agent at a more proximal level into the ICA.
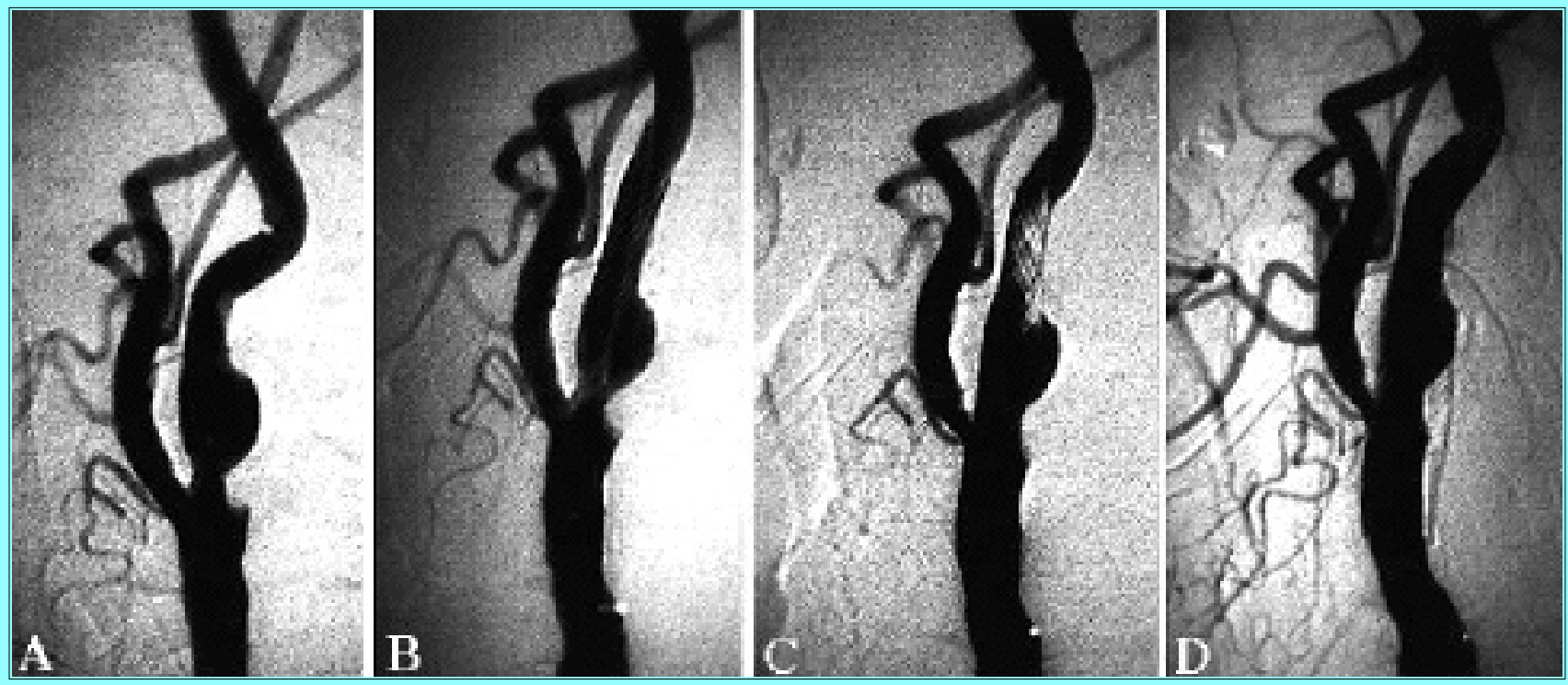

Fig. 3. Angiographic studies illustrating thrombosis of the ICA in the course of stent placement in which there was difficult endovascular access, due to very tortuous supraaortic vessels. No repeated injection of heparin was performed. A: Symptomatic irregular stenosis before treatment. B: Protected placement of a Wallstent without predilation. Note incomplete self-expansion of the stent due to hardness of the plaque. C: Angiogram obtained after protected, complementary angioplasty revealing clotting at the distal extremity of the stent without intracerebral-associated cerebral thromboembolism. The occlusive balloon was reinflated, and 500,000 IU of urokinase was injected into the occluded ICA. After 5 minutes the blood was aspirated and the remaining potential clots were flushed toward the ECA. D: Posttreatment angiogram showing disappearance of the clot.

Embolic Complications Related to Use of Protective Device (Cleaning Procedure). Use of a distal protection balloon, combined with a cleaning procedure in which aspiration and flush injection are performed, may lead also to specific embolic events. We observed two types of embolic events related to the cleaning procedure, both of which occurred during flush application into the freshly stented bifurcation area (Fig. 4). 


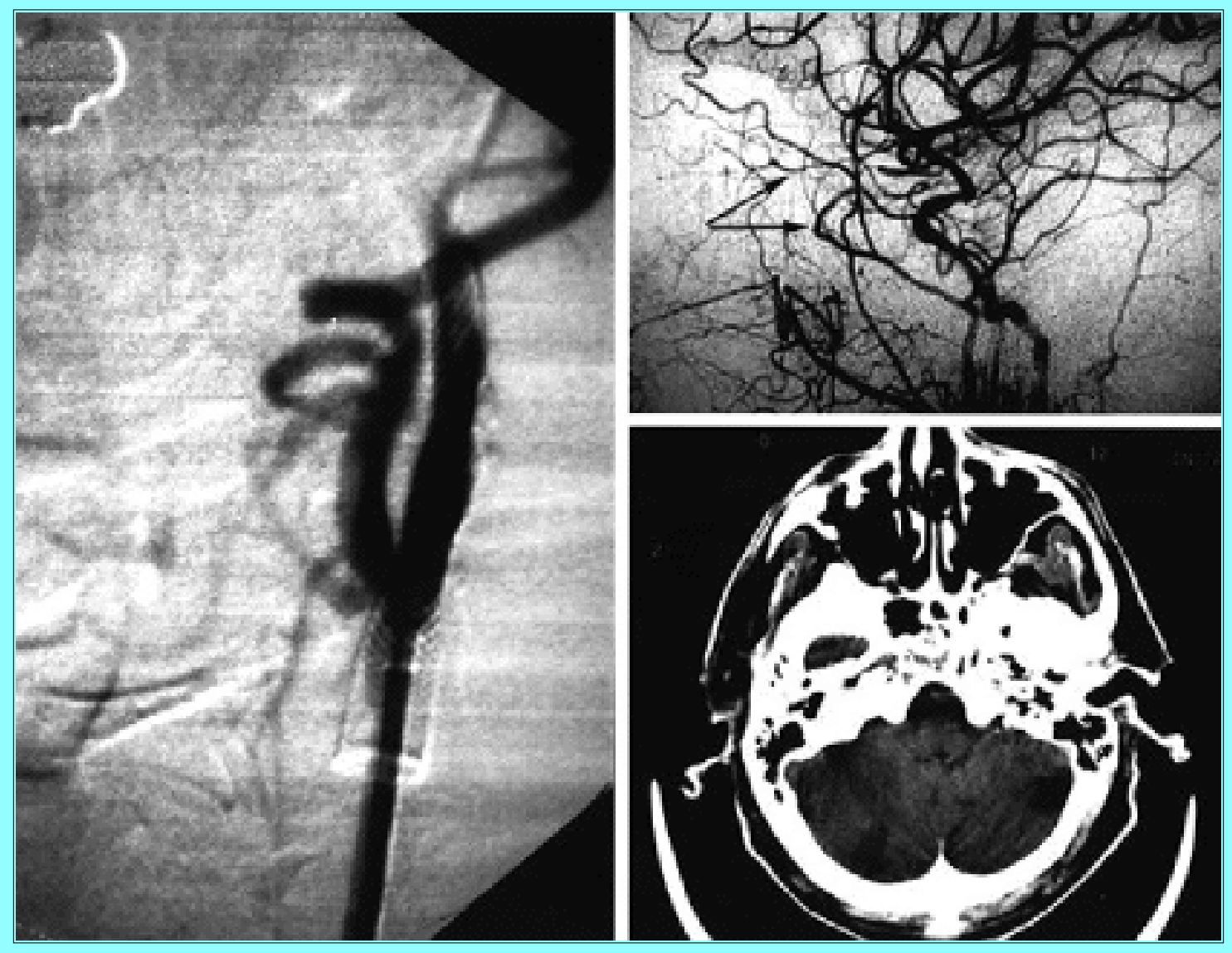

Fig. 4. Diagnostic imaging revealing complications that may occur during cleaning procedure in flushing with protected angioplasty and stenting is used. Left: Angiogram demonstrating temporary occlusion of the ICA. A contrast injection shows the reflux of contrast and potential residual plaque debris toward the ECA. Upper Right: Angiogram revealing a complication related to a normal anatomical variant. In this case, the ophthalmic artery arises from the middle meningeal artery. This variation was overlooked on initial angiographic evaluation, and the patient suffered monocular blindness due to flushing of plaque debris into the ECA territory. Lower Right: Computerized tomography demonstrating areas of small infarctions in the posterior fossa presenting with transient neurological signs. This complication occurred in the course of right carotid artery angioplasty and stenting, while flushing at a $4 \mathrm{ml} / \mathrm{sec}-$ rate for at total of $40 \mathrm{ml}$ flushing fluid injected for cleaning purposes. The rate of injection should not exceed $2 \mathrm{ml} / \mathrm{second}$; beyond that rate there is a risk of reflux toward the vertebral arterial system, particularly on the right side.

After aspiration, the remaining plaque debris was flushed toward an external carotid artery (ECA) territory with so-called dangerous communication. We observed monocular blindness in a patient in whom the presence of an anatomical variant, with supply to the ophthalmic artery territory that came from the middle meningeal artery, was overlooked. It is mandatory to perform and evaluate preliminary diagnositc angiography with visualization of the ECA branches prior to treatment to avoid such events. Other dangerous connections include communicating channels from the internal maxillary artery to the ICA or more frequently from the occipital artery to the vertebral artery. 2) When flushing is too vigorous during the cleaning procedure, it may lead to reflux down to the origin of the carotid artery. In one case this provoked reflux from the right carotid artery toward the right vertebral artery, causing a transiently symptomatic embolization to the vertebrobasilar system. As experimental studies on the model have 
shown, for cleaning with large amounts of fluid, the flush injection should not exceed $2 \mathrm{ml} / \mathrm{second}$ to allow acceptance by the ECA territory. This is more critical on the right side, because the average length of the right CCA is $9.5 \mathrm{~cm}$, whereas the left CCA has an average length of $14 \mathrm{~cm}$.

How to Prevent Embolic Future Events: Device Requirements for Protection Devices. The need to protect patients from embolic plaque debris has initiated the development of specific medical devices by several companies. Principally, devices that block the flow to the ICA or filter the blood during the procedure are currently being developed. In both methods, the retrieval of debris and the cleaning of the treated vascular segment are important technical steps to realize.

\section{Complications of Dissection}

Typical Complication of Dilation Without Stents. Prior to the availability of stents for the carotid artery bifurcation, we observed an incidence of dissection in approximately $5 \%$ of our cases. Dissections were asymptomatic and visible on angiography only or the patient developed neurological symptoms, usually when associated with thrombus formation. Thrombus formation can rapidly occlude the ICA at the cervical level and extend into cerebral arteries (Fig. 5).
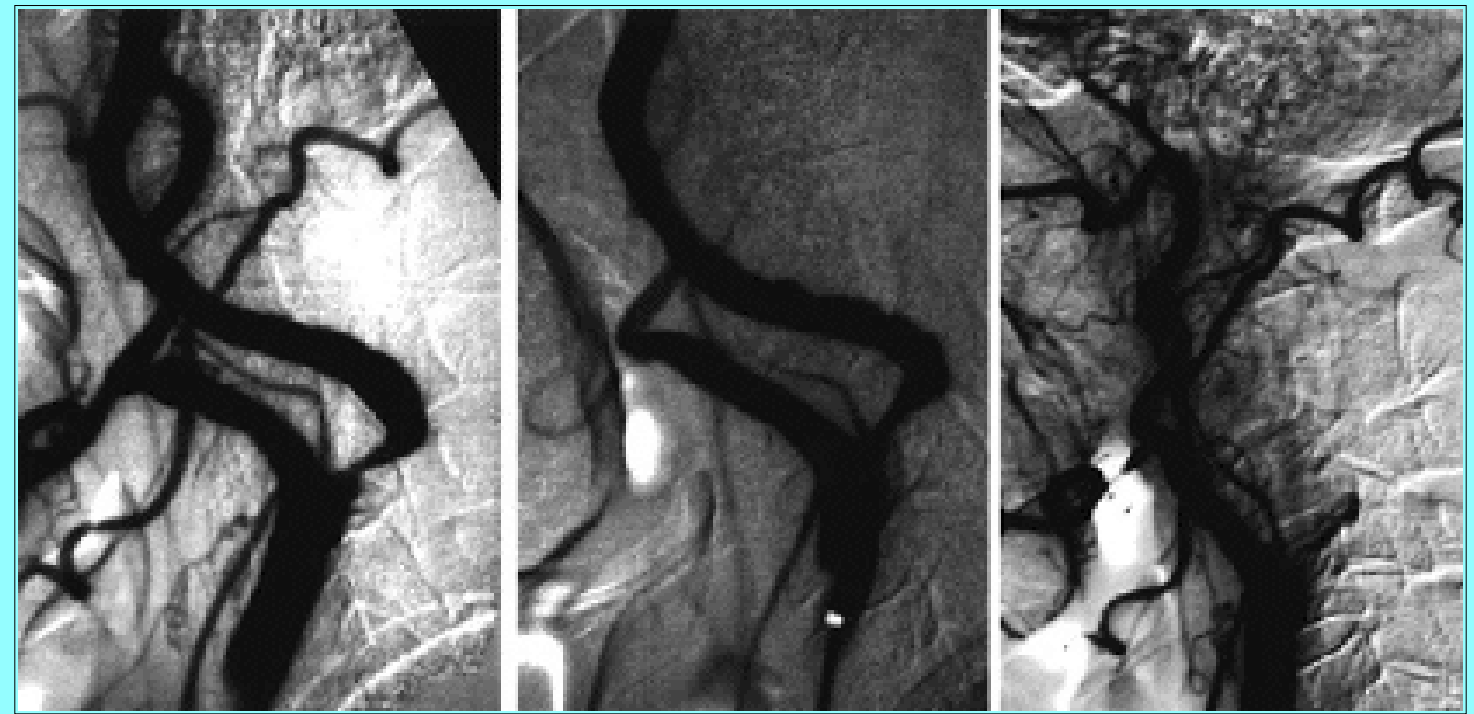

Fig. 5. Angiographic studies. Balloon angioplasty was complicated by a dissection and occlusion of the ICA. Left: Pretreatment angiogram demonstrating narrow stenosis of the ICA. Center: Stenosis was passed with difficulty, producing a false lumen. The control angiogram showed the wrong passage through the plaque. This was not recognized and angioplasty was performed. Right: Angiogram immediately postangioplasty demonstrating occlusion of the ICA.

Such extensive clotting may result in massive hemispheric infarction, a complication we only rarely encountered. When angiography demonstrates endoluminal filling defects that indicate clot formation, one should rapidly increase anticoagulation and thrombolytic drugs, as outlined previously. Based on angiographic studies, the predictive estimation of the risk of dissection is, in our experience, very difficult, and we are unable to provide any angiographically demonstrated plaque pattern of real predictive value (Fig. 6). 

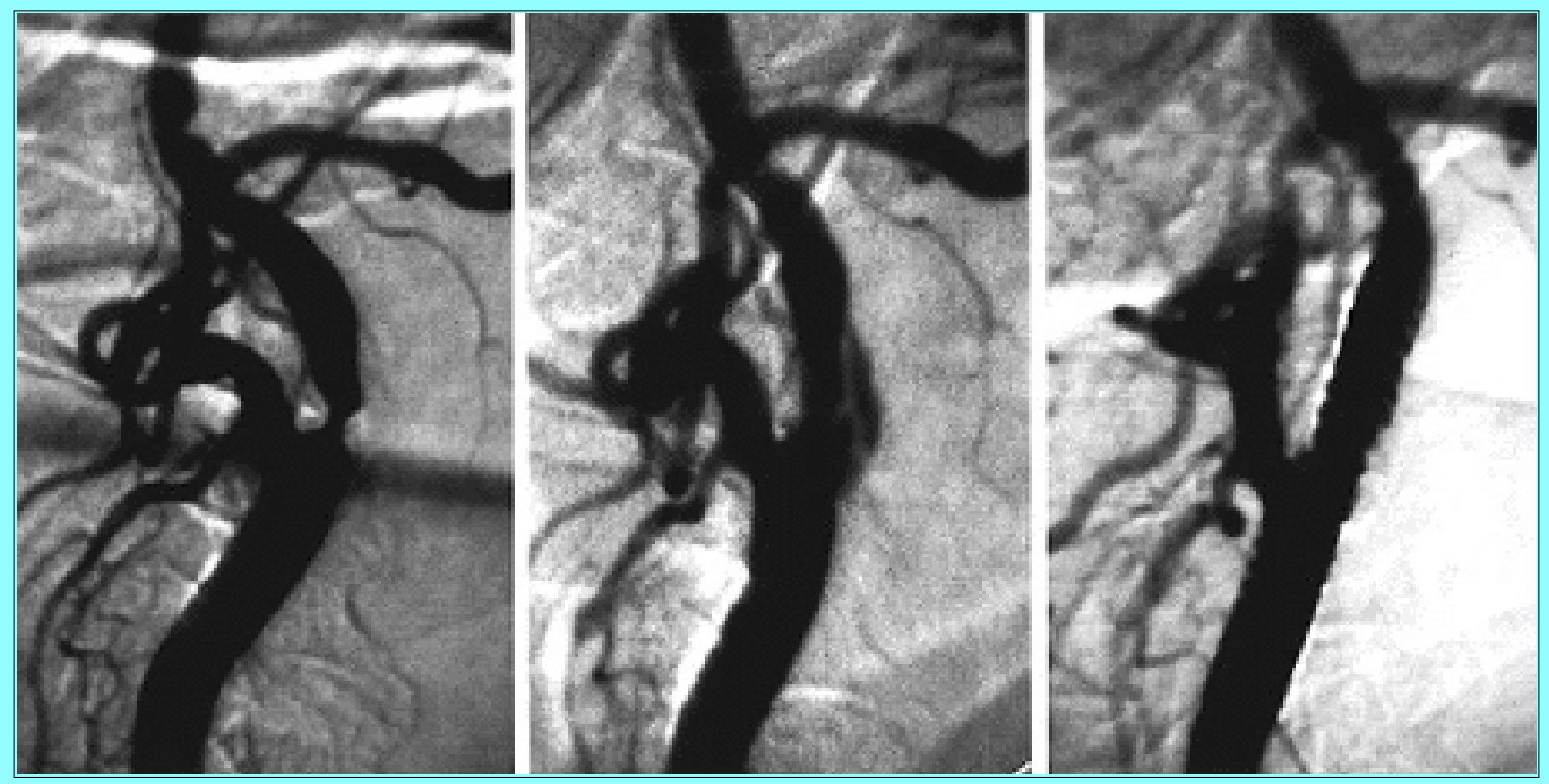

Fig. 6. Angiographic studies illustrating the unpredictable risk of dissection when evaluating variable patterns of stenotic lesions. Left: Angiogram demonstrating apparently short and smooth stenosis. Center: Angiogram obtained after protected angioplasty. The plaque has not been sufficiently compressed, and an obvious subintimal injection is observed. This could have evolved into an occlusion, with extensive intracerebral thrombosis or, perhaps less dramatically, into a lesion with recurrent stenosis. To avert such a complication, a stent was implanted. Right: Angiogram obtained after stent placement of a Strecker stent.

Major Impact of the Stent. In reference to the reported results with current carotid artery stenting performed without protection techniques, stent use had no effect on the risk of observed embolic complications; however, with regard to dissections, the frequency was dramatically reduced. It is due to the capacity of the stents to avert and treat the formation of dissections that a surgical standby is no longer required during carotid artery endovascular recanalization procedures. Stents that cover the whole atheromatous diseased bifurcation have become standard devices mainly because they avoid and treat procedural dissections.

The Remaining Risk of Dissection. Since the introduction of stent use for the treatment of carotid artery lesions, the incidence of dissection has been practically eliminated from the list of potential complications. However, one remaining problem may be the dissection that is not associated with dilation but is related to the difficult first passage through a large plaque. Although seemingly within the arterial lumen, the angioplasty catheter may prove to be outside the lumen and within the plaque, as visualized in a perpendicular second projection (Fig. 7). If such a situation is encountered, we recommend withdrawing the catheter and searching for the real lumen to avoid occlusion of the carotid artery by a dilating a "false" lumen. 

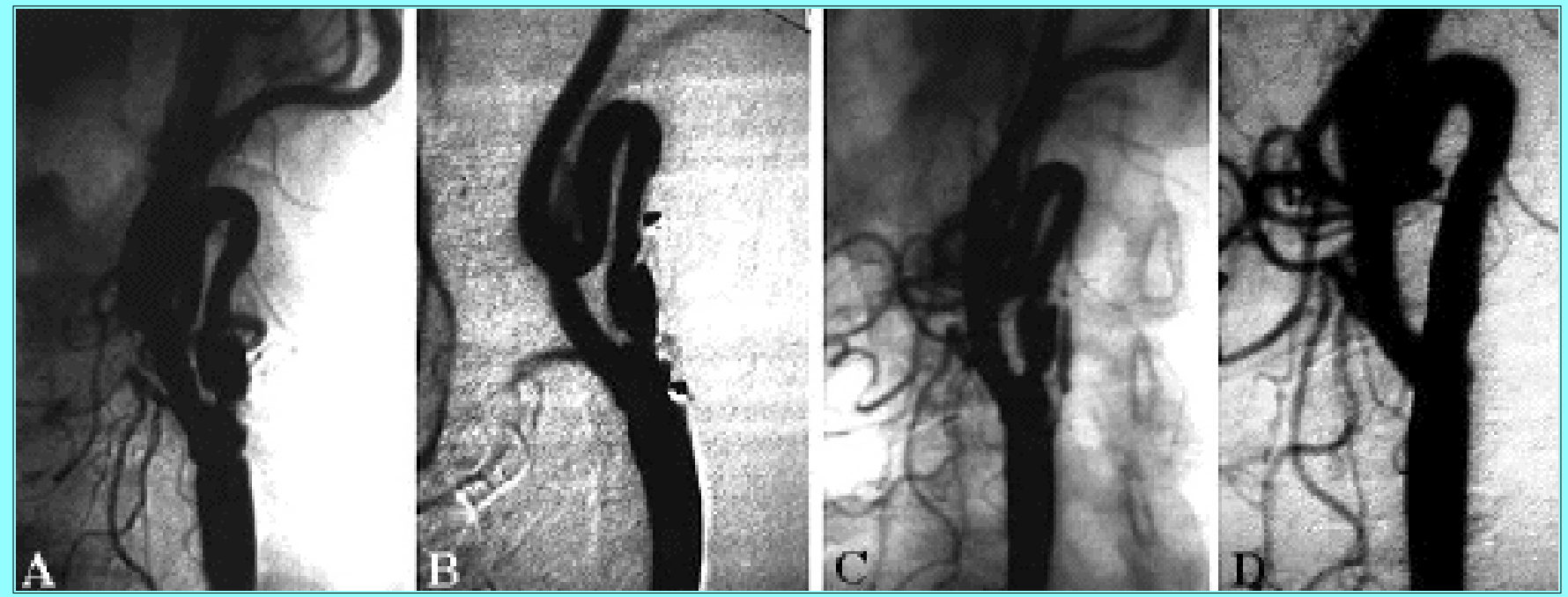

Fig. 7. Angiographic studies exemplifying a potential risk of dissection and occlusion. A: Preangioplasy angiogram demonstrating irregular symptomatic stenosis. B: Angiogram obtained after subintimal passage of the stenosis. Obvious catheter position projecting outside of the arterial lumen. Angioplasty would have led to an occlusion of the artery. C: Angiogram obtained after the angioplasty catheter was withdrawn. Residual subintimal contrast deposit is observed. D: Angiogram obtained after repositioning of the angioplasty catheter into the correct lumen, which show the result of protected angioplasty and stenting.

\section{VASOSPASM}

Why and Where Spasms are Observed. The normal cervical carotid artery above the carotid bulb is known to be quite sensitive to mechanical irritation, producing more or less severe segmental spasm. Mechanical irritation to the normal, nondiseased carotid artery segments that is produced by a guidewire, a stiff dilation balloon, or stent delivery device may induce vasospasm (Fig. 8). Moderate to severe vasospasm may quite frequently be observed when current protection devices are used that irritate the vessel with a balloon. Another source of mechanical irritation can exist when the distal extremity of a stent ends in a vessel curve. To avoid this, we prefer to cover widely such arterial curves by the stent, which induces remodeling,to straighten the cervical segment of the ICA.

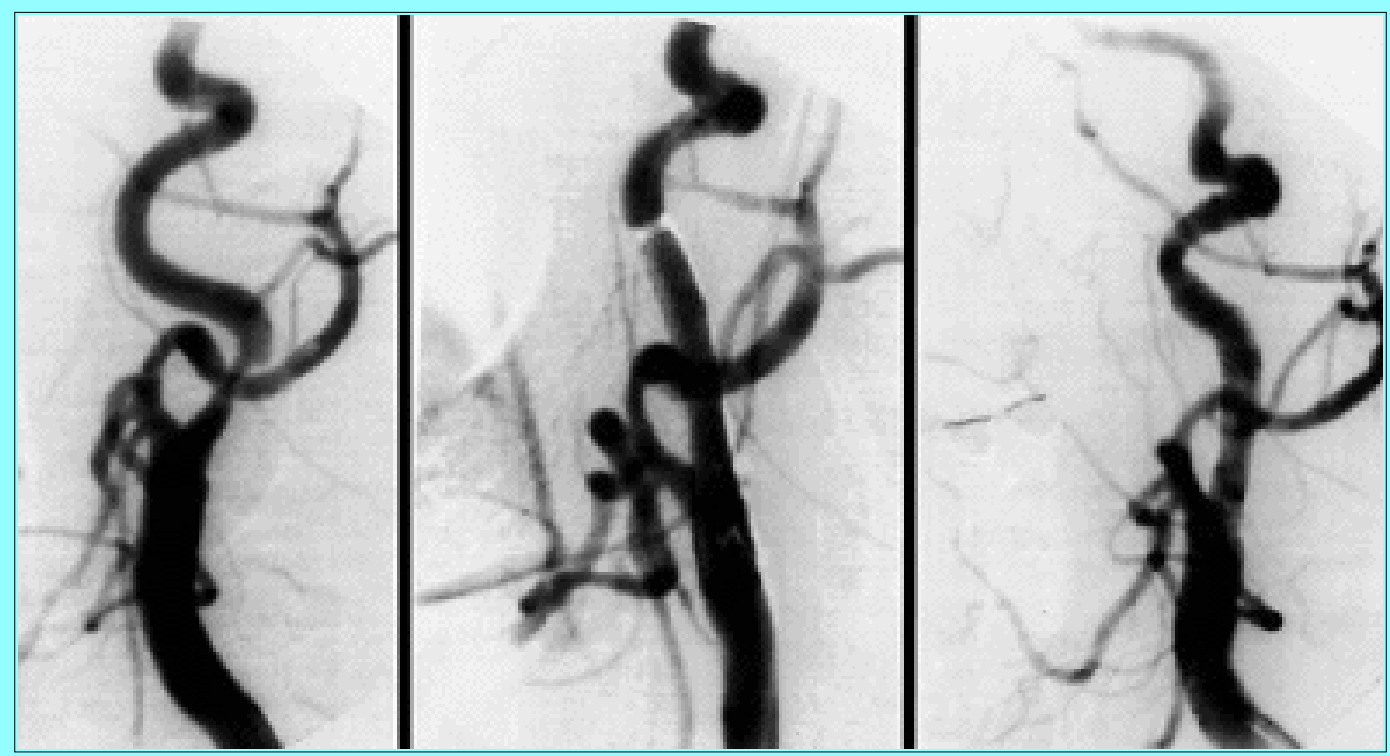

Fig. 8. Angiographic studies illustrating arterial spasm that occurs after protected 
angioplasty and stenting. Left: Pretreatment angiogram revealing stenosis of the ICA with associated tortuousity. Center: Angiogram obtained after protected angioplasty and Wallstent implantation. Note the straightening of the artery and a spasm at the distal extremity of the stent. Right: Angiogram obtained after intraarterial injection of nimodipine exhibiting a significant release of the vasospasm.

How to Treat ICA Vasospasm. If spasm is observed angiographically, we favor active treatment, although only when asymptomatic. Treatment of spasm is medical, using the injectable form of nimodipine (Nimotop) at a dose of $1 \mathrm{ml}(200 \mu \mathrm{g})$ diluted in $10 \mathrm{ml}$ and injected slowly as bolus of 2 to 3 $\mathrm{ml}$ into the carotid artery. The use of nimodipine in treating mechanically induced vasospasm is, in our opinion, efficaccous and quite fast, avoiding further complications such as thrombus formation. With the use of nimodipine, the anesthesiologist must be ready to treat a reduction in mean arterial pressure, because a known side effect of nimodipine is arterial hypotension.

\section{Bradycardia}

Acute-Onset Bradycardia During Dilation and Stent Implantation. Mechanical compression of the carotid artery glomus may produce reactive bradycardia. Such mechanical irritation typically occurs during the balloon dilation period at the carotid artery bifurcation level. Induced bradycardia may result in a reactive, severe hypotension and cause loss of consciousness in a fragile patient.

How to Treat Bradycardia. Bradycardia should be prevented by the intravenous administration of $1 \mathrm{mg}$ of atropine approximately 5 minutes prior to the dilation procedure. If this is forgotten, the anesthesiologist may have to use additional measures to treat the arterial hypotension.

\section{Inappropriate Dilation}

Related to Balloon Use. Early in our experience, an unsatisfactory result for dilation was obtained in a case with a calcified plaque involving both the CCA and ICA, that convinced us to use an oversized balloon system; this resulted in the overdilation of the ICA segment and caused the development of an aneurysm that required secondary surgical repair several months later (Fig. 9). Since the advent of stents that allowed for standard covering of the whole bifurcation area and also for segmental postdilation with use of balloon sizes adapted to the ICA and to the CCA, this type of complication should not occur again. On the other hand, underdilation may lead to restenosis and, even worse, to occlusion.
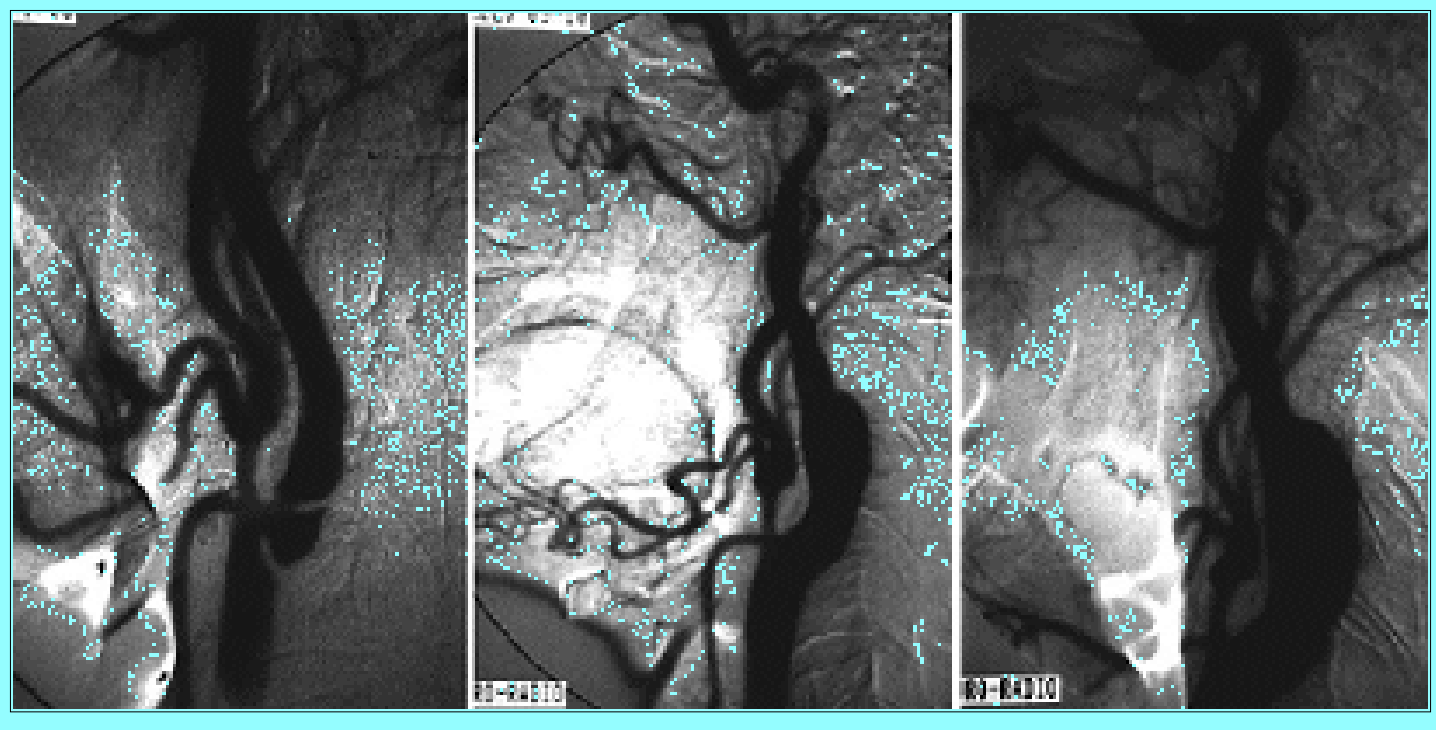
Fig. 9. Angiographic studies illustrating the overdilation of a carotid artery stenosis; this complication occurred before stents were available for use at the carotid artery bifurcation level. Left: Symptomatic ulcerated stenosis. The plaque involved both CCAs and ICAs. With protection, the stenosis was overdilated, mostly in its ICA segment. Center: Angiogram obtained after angioplasty showing how the arterial wall appears smooth with presence of obvious bulging. Right: Follow-up angiogram obtained 6 months later. The patient remained asymptomatic. Note the obvious aneurysm formation of the carotid artery that has been surgically treated with a good result.

Related to Stent Use. In our experience, satisfactory radial force is a critical stent characteristic; because we observed complications related to the use of a weaker "Easy Wallstent" (Schneider), we avoid use of this newer version of stents. The complications we observed were thromboembolic in one patient and locally hemorrhagic in another patient; both occurrences were associated with heavily calcified stenoses. In the first case, the insufficient radial force of this stent type resulted in a partial deployment only, which was constrained by the calcified stenosis. The distal ends of the stent struts persisted to project into the vascular lumen, and this occurred even after considerable postdilation efforts; they served presumably as areas of delayed thrombus formation in a patient who demonstrated a neurological deficit 3 hours after the procedure. The cerebral thromboembolism was treated by local intraarterial thrombolytic therapy. In the other case, the bleeding produced by rupture of a heavily calcified plaque was only stopped after the additional placement of a Palmaz stent. The initially introduced Easy Wallstent did not allow for compression of the injured wall layers, even after repeated postdilation attempts to help better the deployment of the weaker Easy Wallstent (Fig. 10). 


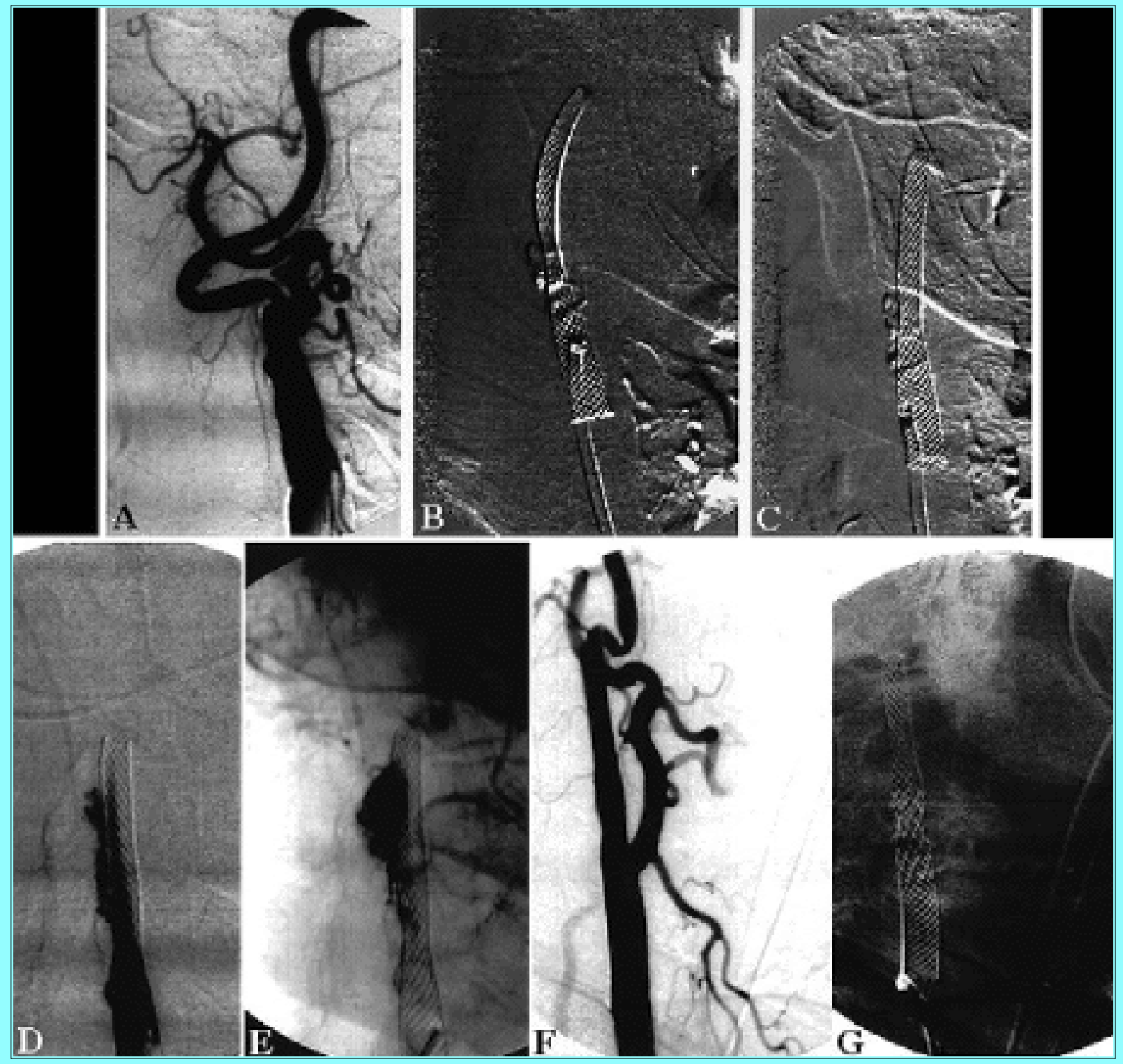

Fig. 10. Angiographic studies exemplifying hemorrhage secondary to stent placement and insufficient radial force of the stent. A: Pretreatment angiogram demonstrating symptomatic stenosis with calcified plaque and very tortuous vessel. B: A Wallstent appears surrounded by the calcifications of the plaque. C: Pattern of the stent after complementary angioplasty showing that the stent remains not fully deployed. D: Early phase study after additional angioplasty demonstrating the blood leaking at the upper part of the calcification. E: Later phase of same study showing the bleeding around the stent. Complementary angioplasty performed using a larger balloon; however, it was unsuccessful. The stent remained incompletely deployed with persistent leak and bleeding. F: A Palmaz stent was placed at the site of the bleeding, allowing for compression of the injured vessel wall structures due to the stronger radial force of this stent. This resulted in bleeding arrest, as demonstrated on the immediately obtained control angiogram. G: Presentation of the stent pattern at the end of the procedure.

Schneider Europe and Boston Scientific Corp., responsible for production of the Easy Wallstent, was informed of these observations that we consider inherent failures. We were informed that a Wallstent version tailored to carotid artery bifurcation treatment and exhibiting the radial force of a "Rolling Membrane" Wallstent with an "Easy" delivery system version will be available soon. Until this carotid 
artery version is available, we will continue to use "Rolling Membrane" Wallstent to treat calcified lesions.

\section{Occlusion of the External Carotid Artery}

Occlusion of the ECA at the origin may occur when a simultaneous severe stenosis of the ECA is not addressed during the procedure for the ICA (Fig. 11). This was rarely observed in our patient group, only when Palmaz or Strecker stents were used, and, in all cases, without clinical symptoms even during the period of prolonged observation. The known, high toleration of ligature of the ECA origin is related to a very efficient collateral supply in this vascular territory.

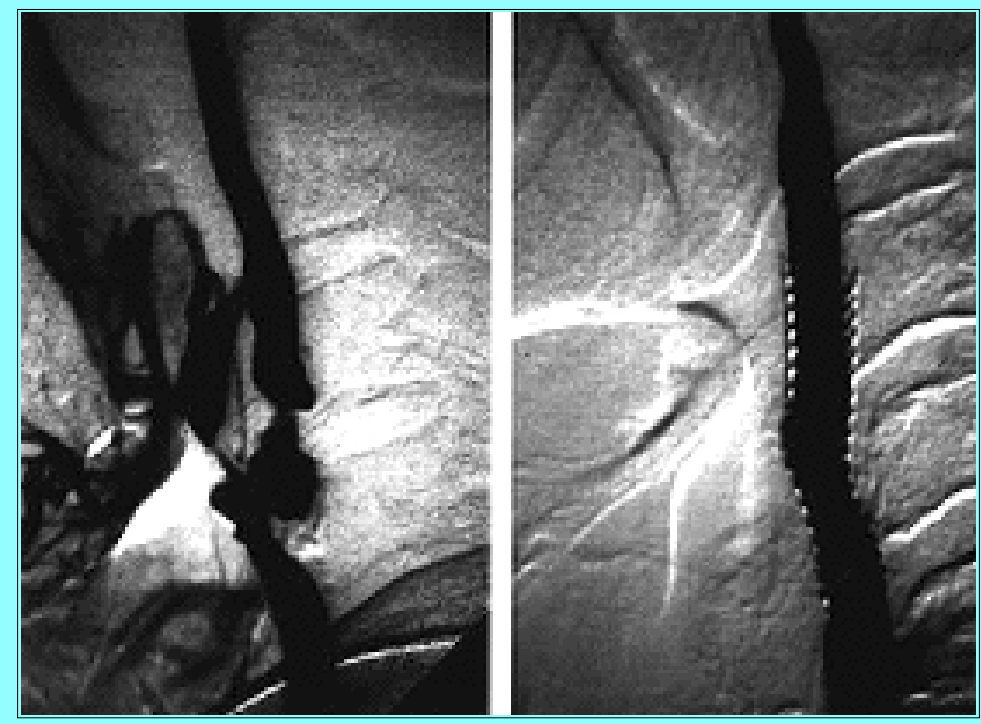

Fig. 11. Angiographic studies illustrating the occlusion of the ECA after stent placement. Left: Symptomatic stenosis of the ICA with preocclusive stenosis of the ECA. Right: Protected angioplasty of the ICA without predilation of the ECA. A Strecker stent was placed. Angiogram, post stent placement, shows a good result in the ICA and an occlusion in the ECA. This occlusion remained asymptomatic.

\section{Unusual Complications}

Although we consider the use of a balloon protection device to be mandatory to avoid embolic complications, additional difficulties may be observed when such devices are used. In one patient in whom satisfactory angioplasty and stent placement for a stenosis of ICA origin was performed, the inflated balloon was inadvertently advanced to the level of the carotid siphon during the flushing procedure, and this resulted in injury of a presumably dysplastic wall of the siphon and causing a cavernous carotido fistula. Detachable platinum coils were used to block this cavernous carotid fistula, and the patient was cured of the complication (Fig. 12). 


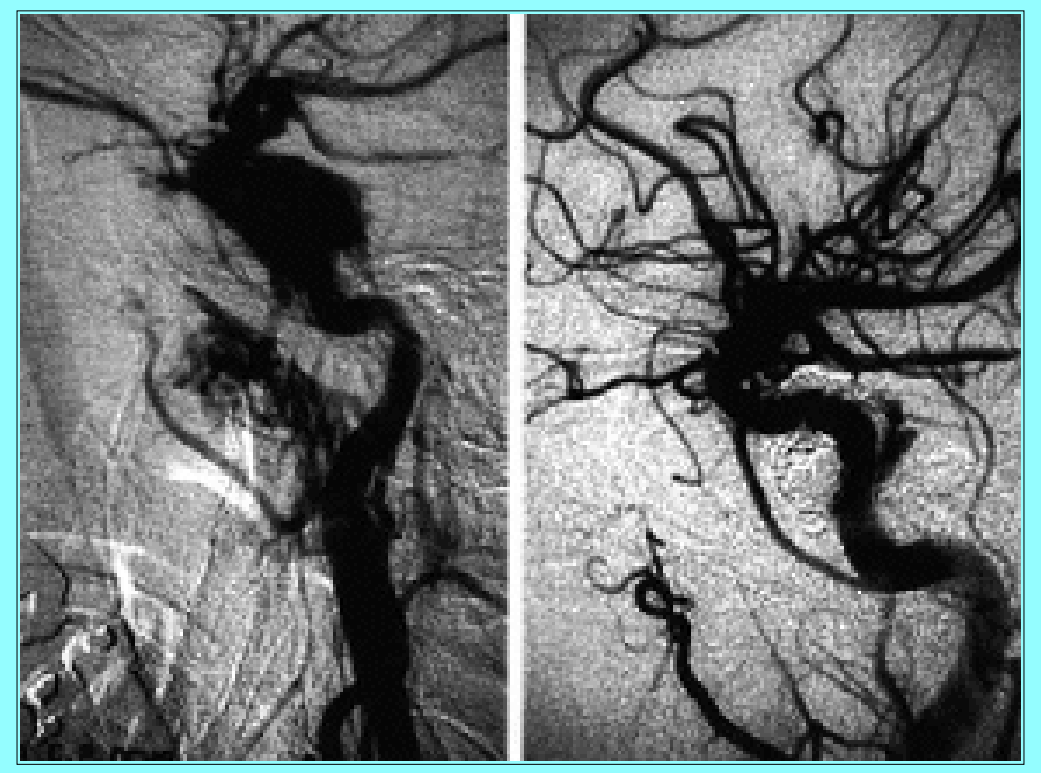

Fig. 12. Angiographic studies demonstrating an unusual complication: a cavernous carotid fistula secondary to the inadvertent migration of the occlusive balloon into a dysplastic carotid artery siphon that occurred during the flushing procedure. Left: Angiogram showing the opacification of the cavernous sinus and superior ophthalmic vein secondary to an injury of the dysplastic carotid siphon by the balloon. Right: Control angiogram demonstrating treatment by detachable coils.

\section{POSTPROCEDURE COMPLICATIONS}

\section{Early Postprocedure Complications}

Why Intensive Care Surveillance is Mandatory After Stent Placement. The advent of the carotid stent has resulted in surgical standby no longer being mandatory, although patient surveillance in an intensive care unit (ICU) remains required. The ICU surveillance, mandatory in the immediate posttreatment period, typically is required overnight for a period of about 12 to 24 hours. The primarily feared complications in the early posttreatment period involve thrombus formation, with occlusion or thromboembolic events, and delayed onset of bradycardia that poses the threat of critical decrease in mean arterial pressure.

\section{Embolic and Occlusive Complications:}

Early posttreatment embolic events after stent placement are basically related to inadequate antiplatelet medication associated with or without an insufficient deployment of the stent. The critical period, when the risk of thromboembolic events in the hours following the procedure is highest, occurs because of decrease in the heparin blood level. Posttreatment surveillance involves frequent neurological checks to determine if any deficits are related to thromboembolic events. In case a new neurological deficit develops or a modification of consciousness occurs, the patient should undergo immediate computerized tomography scanning to rule out cerebral hemorrhage, as well as subsequent control angiography to localize precisely the occlusion and its extent. To provide rapid angiographic access in early posttreatment period is, for us, the most important reason to keep the femoral introducer sheath in place until the following day. Intraarterial thrombolysis will be administered as soon as possible and according to current standards with respect to maximum time delay since symptom onset with or without involvement of the lenticulostriate arteries. 


\section{Hypotension and Bradycardia}

Prolonged Stimulation of Carotid Glomus by the Stent. To prevent hypotension and bradycardia in the early posttreatment period the patient requires continuous surveillance of arterial blood pressure and heart rhythm. A secondary appearance of bradycardia is observed frequently when self-expandable stents are used, which exhibit radial forces similar to that produced by a 8-mm "Rolling Membrane" Wallstent. The use of a stent this size is required to adapt well to the lumen size of both the ICA and CCA and to cover the whole diseased bifurcation area. The occurrence of bradycardia might thus be considered evidence of an efficient and secure deployment of a self-expandable stent with sufficient radial forces. In our experience, the continuous compression of the carotid artery glomus may lead to bradycardia no later than 24 hours postprocedure and medical support provided on the ICU has allowed us to avoid clinical complications related to this side effect of stents. In our ICU, the use of dopamine has assisted us in coping with the effects of delayed bradycardia.

\section{Later Postprocedure Complications}

Restenoses. Restenosis related to myointimal hyperplasia is the most feared long-term complications after endovascular recanalization. In relation to the risk of restenosis at the carotid artery bifurcation, the type of endovascular treatment technique that is applied appears to be critical. In an analysis of patients who underwent angioplasty alone early in our experience, we observed a restenosis rate of 16\% (Fig. 13). Secondary redilation, again performed prior to stent availability, obtained a satisfactory result in $60 \%$ of the cases. Distal protection was not considered necessary when performing redilation procedures, because myoinitimal hyperplasia poses a low risk of producing plaque debris. When detected, restenosis should be treated without delay to avoid formation of a tough scar as lesion that will make redilation procedures more difficult and will often require two sessions.

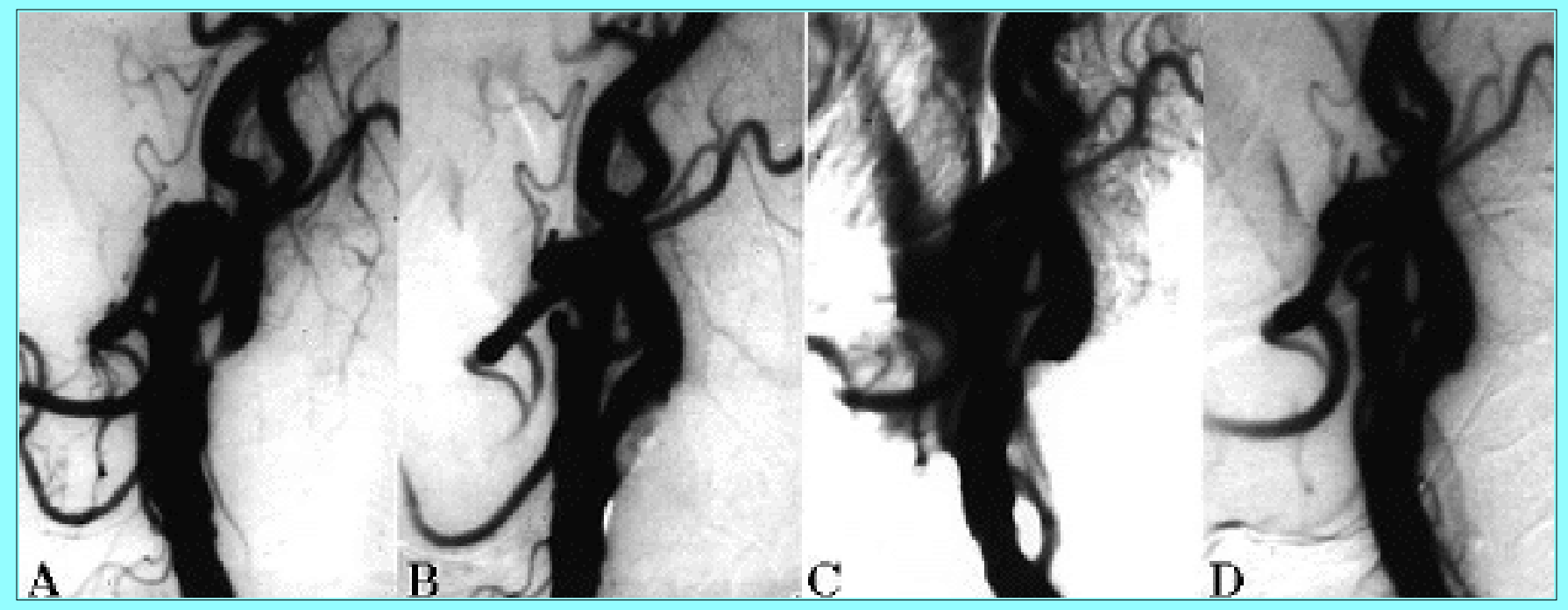

Fig. 13. Angiographic studies illustrating recurrent stenosis after protected angioplasty without stent placement. A: Pretreatment angiogram. Note narrow stenosis of the ICA. B: Angiogram obtained after angioplasty. Suboptimal dilation of the artery due to the hardness of the plaque. At the time of this complication, the stents were not yet available. C:

Follow-up angiogram demonstrating restenosis 6 months later. D: Angiogram showing protected redilation with satisfactory result.

With the advent of stents, the restenosis rate in the carotid artery has dropped significantly from $6 \%$ with the Strecker stent (Fig. 14) to a current low rate of less than $1 \%$ with the Wallstent. Of 145 implanted 
Wallstents, only one case of restenosis presented with asymptomatic myoinitmal hyperplasia. This outcome obviously requires confirmation over a longer follow-up period, and a larger patient population is needed. Nevertheless, this outcome underscores one of the reasons why we currently favor the Wallstent.
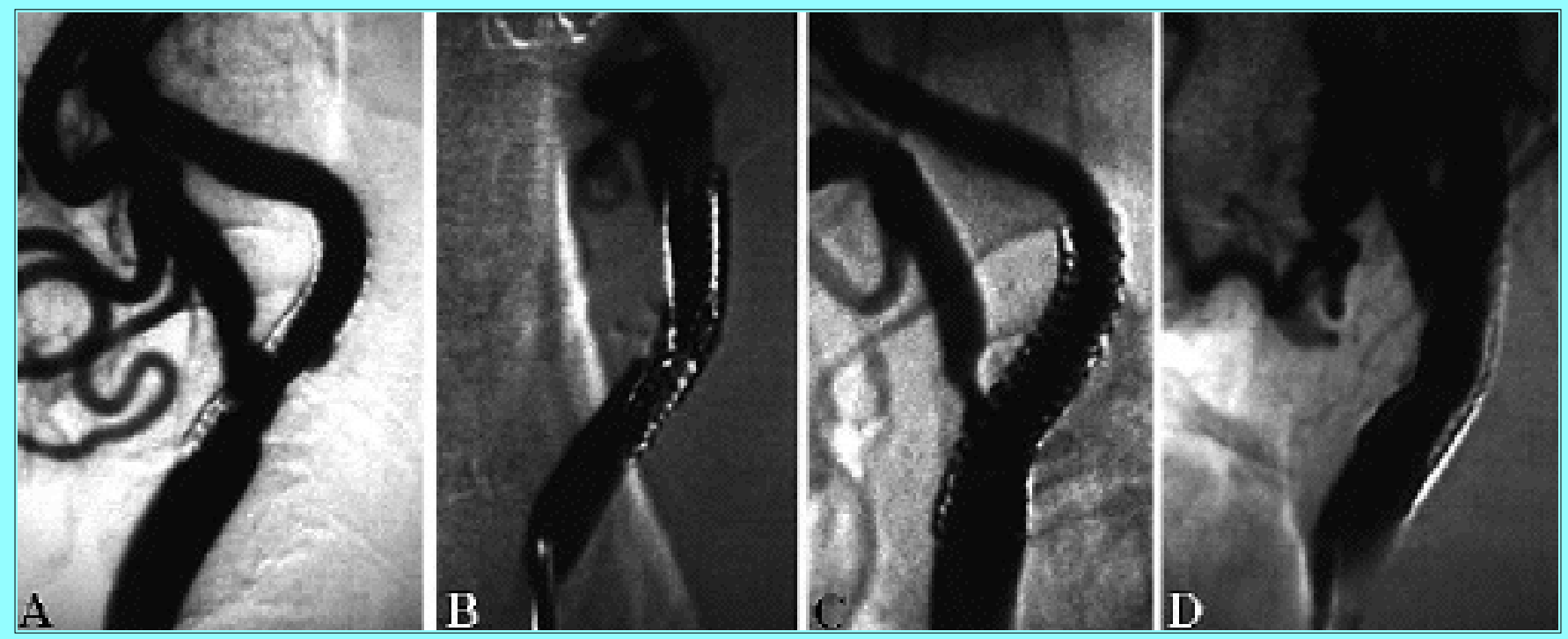

Fig. 14. Angiographic studies demonstrating recurrent stenosis and secondary deformation of a Strecker stent. A: Angiogram, oblique projection, obtained 7 months after stent placement. Note presence of myointimal hyperplasia. B: Angiogram, in anteroposterior projection, revealing myointimal hyperplasia and major deformation of the proximal extremity of the stent only seen in the anteroposterior projection. C: Angiogram, oblique projection, after redilation. D: Angiogram, anteroposterior projection, after redilation.

When treating restenosis that has occurred with implanted stents, we observed a very good response; patients usually required only one session of redilation to obtain a stable and satisfactory result. The process of myointimal hyperplasia seems to be a limited process at the carotid bifurcation level, and we consider early redilation worthwhile if significant restenosis is observed.

Secondary Stent Deformation and Occlusion. Secondary deformation of Palmaz stents used for carotid artery stenting at the bifurcation level have been reported to be associated with the risk of ICA occlusion. We have observed such secondary deformation also when using Strecker stents and have corrected such changes with redilation (Figs. 14 and 15). Secondary stent deformation seems not to be an issue when using of self-expandable stents in the long-term follow-up evaluation, which provides a strong argument in favor of their use, when indicated, in the carotid artery bifurcation. 


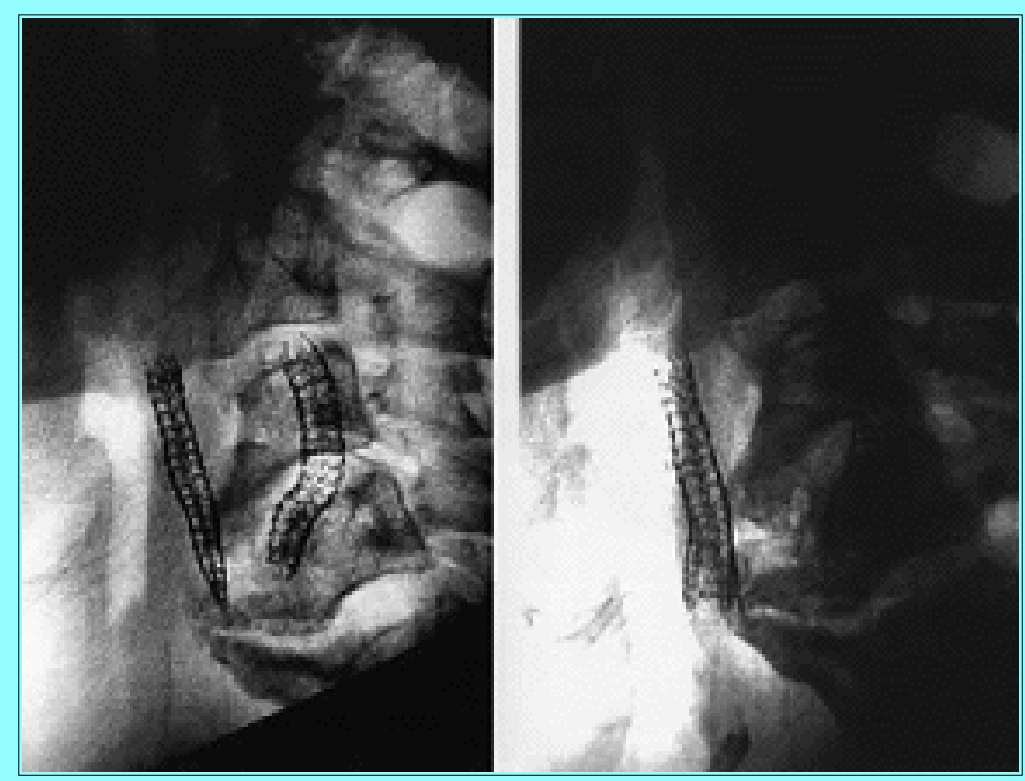

Fig. 15. Angiographic studies illustrating bilateral Stecker stent placement and stent deformation on one side. A: Angiogram showing proximal deformation of the more anterior-projected stent. B: Control angiogram obtained after redilation of the stent.

\section{CONCLUSIONS}

We have reported the complications that occurred in our experience when performing recanalization procedures to treat the ICA, and we have presented treatment suggestions. Descriptions have been provided of how the stenting technique has improved the results and suggestions are provided with regard to further improvement potentially associated with protection device use. Overall, we have demonstrated that adequate use of currently available systems allows for safe application of endovascular treatment methods. A reduced incidence of complications related to the inital individual learning curve may be obtained through preclinical training with in vitro models. Surgical standby no longer seems required; however, early posttreatment surveillance in an ICU is mandatory to avoid the remaining primary complications.

\section{Acknowledgments}

We express our graditude to M. Muster and B. Jean, M.D., for their valuable assistance in the preparation of the image documentation.

\section{References}

1. Brown MM: Balloon angioplasty for cerebrovascular disease. Neurol Res 14(Suppl 12):159-163, 1992

2. Eckert B, Zanella FE, Thie A, et al: Angioplasty of the internal carotid artery: results, complications and follow-up in 61 cases. Cerebrovasc Dis 6:97-105, 1996 (Reference unverified)

3. Mathias K: Katheterbehandlung der arteriellen Verschlusskrankheit supraaortaler Gefässe. Radiologe 27:547-554, 1987

4. Munari LM, Belloni G, Peretti A, et al: Carotid percutaneous angioplasty. Neurol Res 14 (Suppl 2):156-158, 1992 
5. Ohki T, Marin ML, Lyon RT, et al: Ex vivo human carotid artery bifurcation stenting: correlation of lesion characteristics with embolic potential. J Vasc Surg 27:463-471, 1998

6. Roubin GS, Yadav S, Lyer SS, et al: Carotid stent-supported angioplasty: a neurovascular intervention to prevent stroke. Am J Cardiol 78:8-12, 1996

7. Théron J, Courtheoux P, Alachkar F, et al: New triple coaxial catheter system for carotid angioplasty with cerebral protection. AJNR 11:869-877, 1990

8. Théron JG, Payelle GG, Coskun O, et al: Carotid artery stenosis: treatment with protected balloon angioplasty and stent placement. Radiology 201:627-636, 1996

9. Tsao FY, Higashida R, Meoli C: Percutaneous transluminal angioplasty of extracranial and intracranial arterial stenosis in the head and neck. Neuroimaging Clin North Am 2:371-384, 1992 (Reference unverified)

Manuscript received November 2, 1998.

Accepted in final form November 18, 1998.

Accepted at the International Society of Carotid Artery Therapy (ISCAT 1998).

Address reprint requests to: Martin Jean Baptiste, M.D., Neuroradiology Department, University Hospital of Geneva, 24 Michael DuCrest, 1211 Geneva, Switzerland. email: jean.martin@medecine.unige.ch. 